\title{
MedActive Oral Product
}

National Cancer Institute

\section{Source}

National Cancer Institute. MedActive Oral Product. NCI Thesaurus. Code $C 95197$.

Proprietary, over-the-counter products containing stannous fluoride and Spilanthes extract for the relief of dry mouth symptoms. 